This is the peer reviewed version of the following article: [Grandi, S.M., Vallée-Pouliot, K., Reynier, P., Eberg, M., Platt, R.W., Arel, R., Basso, O., and Filion, K.B. (2017). Hypertensive Disorders in Pregnancy and the Risk of Subsequent Cardiovascular

\title{
Hypertensive Disorders in Pregnancy and the Risk of Subsequent Cardiovascular Disease
}

\author{
Sonia M. Grandi, ${ }^{a}$ Karine Vallée-Pouliot, ${ }^{a}$ Pauline Reynier, ${ }^{\mathrm{b}}$ Maria Eberg, ${ }^{\mathrm{b}}$
} Robert W. Platt, ${ }^{a, c, d}$ Roxane Arel, ${ }^{e}$ Olga Basso, ${ }^{a, c, f}$ Kristian B. Filion ${ }^{a, b, g}$

\section{Author affiliations}

a. Department of Epidemiology, Biostatistics and Occupational Health, McGill University, Montreal, QC, Canada

b. Centre for Clinical Epidemiology, Lady Davis Institute, Jewish General Hospital, Montreal, QC, Canada

c. McGill University Health Center Research Institute, Montreal, QC, Canada

d. Department of Pediatrics, McGill University, Montreal, QC, Canada

e. Department of Family Medicine, St. Mary's Hospital Centre, McGill University, Montreal, QC, Canada

f. Department of Obstetrics and Gynecology, McGill University, Montreal, QC, Canada

g. Department of Medicine, McGill University, Montreal, QC, Canada

\section{Address for Correspondence}

Kristian B. Filion

Departments of Medicine and of Epidemiology, Biostatistics, and Occupational Health McGill University

Centre for Clinical Epidemiology

Montreal, Quebec, Canada

Email: kristian.filion@mcgill.ca

Word Count (Text): 3,496

Word Count (Abstract): 242 
This is the peer reviewed version of the following article: [Grandi, S.M., Vallée-Pouliot, K., Reynier, P., Eberg, M., Platt, R.W., Arel, R., Basso, O., and Filion, K.B. (2017). Hypertensive Disorders in Pregnancy and the Risk of Subsequent Cardiovascular

Short Title: Hypertensive Disorders and Risk of Cardiovascular Disease 
This is the peer reviewed version of the following article: [Grandi, S.M., Vallée-Pouliot, K., Reynier, P., Eberg, M., Platt, R.W., Arel, R., Basso, O., and Filion, K.B. (2017). Hypertensive Disorders in Pregnancy and the Risk of Subsequent Cardiovascular

\section{ABSTRACT}

Background: Hypertensive disorders in pregnancy (HDP) have been shown to predict later risk of cardiovascular disease (CVD). However, previous studies have not accounted for subsequent pregnancies and their complications, which are potential confounders and intermediates of this association.

Methods: A cohort of 146,748 women with a first pregnancy was constructed using the Clinical Practice Research Datalink. HDP was defined using diagnostic codes, elevated blood pressure readings, or new use of an anti-hypertensive drug between 18 weeks' gestation and 6 weeks postpartum. The study outcomes were incident CVD and hypertension. Marginal structural Cox models (MSM) were used to account for time-varying confounders and intermediates. Timefixed exposure defined at the first pregnancy was used in secondary analyses.

Results: A total of 997 women were diagnosed with incident CVD, and 6,812 women were diagnosed with hypertension or received a new anti-hypertensive medication during the followup period. Compared with women without HDP, those with HDP had a substantially higher rate of CVD (HR 2.2, 95\% Cl 1.7, 2.7). In women with HDP, the rate of hypertension was 5 times that of women without a HDP (HR 5.6, 95\% Cl 5.1,6.3). With overlapping 95\% Cls, the time-fixed analysis and the MSM produced consistent results for both outcomes.

Conclusions: Women with HDP are at increased risk of developing subsequent CVD and hypertension. Similar estimates obtained with the MSM and the time-fixed analysis suggests that subsequent pregnancies do not confound a first episode of HDP and later CVD.

Key words[CVA1]: Hypertensive disorders in pregnancy, cardiovascular disease, hypertension, pregnancy, marginal structural model. 
This is the peer reviewed version of the following article: [Grandi, S.M., Vallée-Pouliot, K., Reynier, P., Eberg, M., Platt, R.W., Arel, R., Basso, O., and Filion, K.B. (2017). Hypertensive Disorders in Pregnancy and the Risk of Subsequent Cardiovascular

\section{INTRODUCTION}

Over the last decade, the incidence of hypertensive disorders in pregnancy (HDP) has steadily increased. ${ }^{1}$ Several studies have reported an increased risk of cardiovascular disease (CVD) following preeclampsia, and more recent evidence suggests that all HDP appear to be associated with later CVD. ${ }^{2-5}$ Based on its pathophysiology, it is biologically plausible that preeclampsia is associated with long-term risk of CVD. ${ }^{6}$ Although different pathologies are believed to cause gestational hypertension and preeclampsia, the magnitude of the association of all HDP with subsequent CVD remains unclear, as these conditions are often paired with other known CVD risk factors. ${ }^{2}$ Moreover, previous studies examining the association between HDP and subsequent CVD did not effectively account for subsequent pregnancies and their complications, both of which are potential confounders and intermediates in this association. ${ }^{7-9}$ Furthermore, with long follow-up periods, adjustment for only baseline covariates may result in important residual confounding. The objective of this study was therefore to assess the association between HDP and the risk of incident CVD, incorporating multiple pregnancies and adjusting for time-varying confounding by intermediate variables.

\section{METHODS}

\section{Data Source}

We carried out a population-based cohort study using data extracted from the United Kingdom's Clinical Practice Research Datalink (CPRD). The CPRD is a clinical database that contains the records of over 13 million patients seen at more than 674 general practitioner (GP) practices, and has been shown to be a representative sample of the UK population. ${ }^{10}$ In the UK, 
This is the peer reviewed version of the following article: [Grandi, S.M., Vallée-Pouliot, K., Reynier, P., Eberg, M., Platt, R.W., Arel, R., Basso, O., and Filion, K.B. (2017). Hypertensive Disorders in Pregnancy and the Risk of Subsequent Cardiovascular

specialists and other healthcare providers are required to report back to the GP, who serves as the gatekeeper to the healthcare system. ${ }^{11}$ The CPRD includes clinical diagnoses and referrals to specialists, with diagnoses recorded using Read codes, ${ }^{11}$ a hierarchical coding system that contains $>80,000$ terms to aid in capturing the many dimensions of a patient's state of health. ${ }^{12}$ In addition, all prescriptions issued by GPs are automatically recorded in the CPRD and classified according to the British National Formulary. The CPRD also contains clinical measures such as blood pressure, laboratory tests, and lifestyle variables (e.g., smoking, body mass index [BMI], alcohol use) not commonly found in administrative databases. This study was approved by the research ethics board of the Jewish General Hospital and by the Independent Scientific Advisory Committee of the CPRD (protocol 14_177A2).

\section{Study Population}

In the CPRD, we identified all women between 15 to 45 years of age with a first recorded delivery between January 1990 and December 2013 using Read codes. The date of cohort entry was defined as 42 days after the delivery date, to avoid the period during which symptoms of HDP (and corresponding medication use) may persist, ${ }^{13,14}$ and to avoid misclassifying exposure as an outcome. Inclusion was restricted to women with $\geq 2$ years of observation time in the CPRD prior to cohort entry to allow for the assessment of previous medical (including obstetric) history and for the measurement of potential confounders. Based on prior work on the accuracy of estimation of gestational age in the CPRD, ${ }^{15}$ we assigned deliveries with a record of a term delivery a gestational age of 40 weeks (280 days), and deliveries with a record of a

preterm delivery a gestational age of 35 weeks ( 245 days). ${ }^{16,17}$ 
This is the peer reviewed version of the following article: [Grandi, S.M., Vallée-Pouliot, K., Reynier, P., Eberg, M., Platt, R.W., Arel, R., Basso, O., and Filion, K.B. (2017). Hypertensive Disorders in Pregnancy and the Risk of Subsequent Cardiovascular

Women were excluded if they: (i) had a record of a previous delivery; (ii) had a diagnosis of hypertension before 18 weeks of gestation for the first pregnancy; (iii) had a history of CVD (see Online Appendix 1 for a detailed definition) prior to cohort entry; (iv) had $\geq 2$ measures of systolic blood pressure (SBP) $\geq 140 \mathrm{mmHg}$ or diastolic blood pressure (DBP) $\geq 90 \mathrm{mmHg}$ prior to 18 weeks of gestation; (v) had a DBP of $\geq 110 \mathrm{mmHg}$ prior to 18 weeks of gestation; (vi) were younger than 15 or older than 45 years at first pregnancy; and (vii) used an anti-hypertensive medication before 18 weeks of gestation (see Online Appendix 1).

Women were followed until an event (incident CVD or hypertension) or censoring due to end of CPRD registration, last data collection, or end of the study period (December $31^{\text {st }}, 2013$ ), whichever occurred first. For CVD, women with a pregnancy unaffected by HDP were also censored if: (i) a diagnostic code for hypertension appeared outside of the exposure assessment period; or (ii) a recorded prescription of an anti-hypertensive medication appeared outside of the exposure assessment period. Since new diagnoses of hypertension and new use of anti-hypertensive drugs are included as part of the exposure definition, women with existing hypertension were no longer considered at risk for exposure in subsequent pregnancies. We accounted for such informative censoring in our analysis using inverse probability weights. These women were not censored in our hypertension analyses, as they met our endpoint definition. For both outcomes, unexposed women were censored if they had a diagnostic code for HDP appearing outside of the exposure assessment period. 
This is the peer reviewed version of the following article: [Grandi, S.M., Vallée-Pouliot, K., Reynier, P., Eberg, M., Platt, R.W., Arel, R., Basso, O., and Filion, K.B. (2017). Hypertensive Disorders in Pregnancy and the Risk of Subsequent Cardiovascular

\section{Hypertensive disorders of pregnancy}

We used a time-dependent definition for HDP in which women with HDP in any pregnancy were considered to be exposed as of the date of diagnosis and those with no HDP in any pregnancy were considered to be unexposed. Women meeting any of the following 5 criteria (measured between 18 weeks' gestation and 6 weeks post-delivery) were considered to be exposed: (i) a diagnosis of HDP, including gestational hypertension, preeclampsia (mild, moderate, and severe), eclampsia, hypertension complicating pregnancy, toxaemia, transient hypertension in pregnancy, benign essential hypertension in pregnancy, and hypertension complicated by proteinuria; (ii) a new diagnosis of hypertension in women with normal blood pressure prior to 18 weeks' gestation; (iii) $\geq 2$ consecutive measures of SBP $\geq 140 \mathrm{mmHg}$ or DBP of $\geq 90 \mathrm{mmHg}$; (iv) a first DBP reading $\geq 110 \mathrm{mmHg}$; or (v) new use of an anti-hypertensive medication . For the classification of exposure, we used a 2-week grace period (18 weeks instead of 20 weeks) to account for possible misclassification of gestational age based on our imputation. In secondary analyses, exposure was sub-classified hierarchically, using 3 mutuallyexclusive, time-dependent categories: (i) preeclampsia or eclampsia[CVA2] (with or without other HDP); (ii) other HDP (including elevated blood pressure or use of anti-hypertensive medications); (iii) no HDP. In sensitivity analyses, we used a time-fixed approach, in which exposure was defined based on the first pregnancy.

\section{Outcome definition}

We defined the primary outcome of incident CVD, based on Read codes, as any diagnosis of cerebrovascular disease, coronary artery disease, coronary revascularization, myocardial 
This is the peer reviewed version of the following article: [Grandi, S.M., Vallée-Pouliot, K., Reynier, P., Eberg, M., Platt, R.W., Arel, R., Basso, O., and Filion, K.B. (2017). Hypertensive Disorders in Pregnancy and the Risk of Subsequent Cardiovascular

infarction, peripheral arterial disease, transient ischemic attack, and stroke. The secondary outcome of newly-diagnosed hypertension was defined as a composite of a new diagnosis of hypertension or a new use of an anti-hypertensive drug outside of the exposure assessment period. Anti-hypertensive medications included angiotensin-converting-enzyme (ACE) inhibitors, angiotensin II receptor antagonists (ARBs), beta-blockers, calcium channel blockers, and diuretics. The date of diagnosis or prescription that first met our event definition defined the event date.

\section{Potential confounders}

Potential confounders in the analysis were based on clinical expertise and previous literature supporting their association with the exposure and outcome. We considered both time-fixed and time-varying confounders. The baseline comorbidities were measured any time before start of the first pregnancy, and medication use was assessed in the year before. Baseline timefixed covariates included: maternal age (5-year categories), multifetal gestation (in the index pregnancy), smoking (measures taken up to 5 years prior to the start of the first pregnancy up until the time to delivery), obesity (BMI measures taken up to 5 years prior to the start of the first pregnancy), excessive alcohol use [measures taken up to 5 years prior to the start of the first pregnancy up until the time to delivery; defined using diagnostic codes for excess alcohol use, alcohol-related comorbidities (e.g., cirrhosis), or treatments for alcohol abuse], year of cohort entry ( $\leq 1995,1996-1999,2000-2005$, and $\geq 2006)$, region of residence (a proxy for socioeconomic status and access to care), history of depression, dyslipidemia, venous thromboembolism, polycystic ovary syndrome, gestational diabetes (defined as ever/never and 
This is the peer reviewed version of the following article: [Grandi, S.M., Vallée-Pouliot, K., Reynier, P., Eberg, M., Platt, R.W., Arel, R., Basso, O., and Filion, K.B. (2017). Hypertensive Disorders in Pregnancy and the Risk of Subsequent Cardiovascular

measured from 12 weeks' gestation to 6 weeks postpartum), diabetes mellitus, renal disease, family history of hypertension, family history of CVD, number of distinct drug classes (0-1, 2-4, and $>4$ ), and use of statins, aspirin, and antidepressants (see Online Appendix 1 for detailed list).

Time-dependent variables included: obesity, number of live births during follow-up (1, 2-4, and $\geq 5$ ), multifetal gestation (defined as ever or never), gestational diabetes, and use of aspirin, statin, and anti-depressants. Of the time-dependent variables, number of live births, and use of aspirin and statins were considered as potential intermediates in the relationship of interest (Online Appendix 2). For women with multiple deliveries, outcomes of previous pregnancies[CVA3] were considered as potential confounders for the current pregnancy in our primary analysis. Time-varying confounders were updated every 6 months during follow-up. For the outcome of CVD, we adjusted for history of sleep apnea and migraines at baseline, and use of oral contraceptives, non-steroidal anti-inflammatory drugs, warfarin, and anti-migraine medications. For the time-dependent medication variables, a woman was considered to be currently using that medication if the prescription was issued during the current 6-month interval. For BMI, only measurements taken a year before and a year after a recorded delivery were included to avoid misclassification due to pregnancy-related weight gain. If no BMI values were available for five years, we considered the value to be missing. Missing data for smoking and BMI were imputed using the multiple imputation by chained equations method[CVA4] with 5 imputations. $^{18}$ 
This is the peer reviewed version of the following article: [Grandi, S.M., Vallée-Pouliot, K., Reynier, P., Eberg, M., Platt, R.W., Arel, R., Basso, O., and Filion, K.B. (2017). Hypertensive Disorders in Pregnancy and the Risk of Subsequent Cardiovascular

\section{Statistical Analysis}

We applied a weighted, pooled, logistic regression model to estimate the parameters of the marginal structural Cox model (MSM).${ }^{19}$ Inverse probability-of-treatment and censoring weights were used to account for time-dependent confounding, losses-to-follow-up, and informative censoring. Weights were calculated as the product of two factors: (i) the inverse of a patient's probability of having the exposure history she actually had; and (ii) the inverse of a patient's probability of remaining uncensored. ${ }^{19}$ The weights were stabilized to improve the precision of the estimates. In the weighted pooled logistic model, the time-dependent intercept was estimated using a polynomial function of time or a smoothing function, based on the time since cohort entry, using natural cubic splines.

We then carried out an analysis using a Cox proportional hazards model in which exposure was defined by the first pregnancy and included only baseline covariates. This time-fixed approach (or first pregnancy approach) has known limitations, including the inability to account for subsequent pregnancies or the number of previous pregnancies with HDP, both of which are time-dependent confounders and intermediates. Furthermore, with up to 24 years of followup, adjustment for baseline covariates only may result in substantial residual confounding. Despite these limitations, we included this analysis, to assess the magnitude of time-dependent confounding on the association under investigation. The time scale in the Cox models for the primary and secondary analyses was follow-up time since cohort entry.

\section{Sensitivity Analyses}


This is the peer reviewed version of the following article: [Grandi, S.M., Vallée-Pouliot, K., Reynier, P., Eberg, M., Platt, R.W., Arel, R., Basso, O., and Filion, K.B. (2017). Hypertensive Disorders in Pregnancy and the Risk of Subsequent Cardiovascular

In the main sensitivity analyses, we further sub-classified exposure, separating women with preeclampsia and eclampsia from those with other HDP. We then examined whether the exclusion of women taking cardiovascular medications (including any combination of statins, warfarin, fibrates, and aspirin) in the year prior to cohort entry influenced the association of interest. The impact of varying the exposure assessment window from 6 to 12 weeks postpartum was also examined, as symptoms related to HDP may persist up to 8 weeks postpartum. ${ }^{20}$ In addition, we used age as the time scale in our Cox models. Finally, we compared the association of HDP in a first versus a later pregnancy by restricting the cohort to women with at least two pregnancies and a first unaffected pregnancy.

\section{$\operatorname{RESULTS}_{[\mathrm{CVA5}]}$}

Our study cohort included 146,748 women who had at least one recorded delivery from 1990 to 2013 (Figure 1). The median length of follow-up was 4.7 years (IQR 1.9, 9.1) for our primary outcome of CVD and 4.4 years (IQR $1.8,8.8)$ for our secondary outcome of hypertension. Twothirds of women had 1 pregnancy (69.4\%), a third of women had 2 pregnancies $(30.1 \%)$, and $<1 \%$ of women had three or more pregnancies (0.5\%; Online Appendix 3 ) during the study period. During the follow-up, $1.8 \%(n=6,433)$ of women had 1 pregnancy affected by HDP and $0.6 \%(n=421)$ of women had $\geq 2$ pregnancies affected by HDP (Online Appendix 4A). The majority of women did not have a pregnancy affected by HDP during the study period (95.6\%; $n=140,315)$. 
This is the peer reviewed version of the following article: [Grandi, S.M., Vallée-Pouliot, K., Reynier, P., Eberg, M., Platt, R.W., Arel, R., Basso, O., and Filion, K.B. (2017). Hypertensive Disorders in Pregnancy and the Risk of Subsequent Cardiovascular

At the first recorded pregnancy, the mean age of women was 29.5 (SD 6.1) years, and the majority had a singleton birth (Table 1). Women with HDP were more likely to be overweight or obese, to have diabetes mellitus, gestational diabetes in their first pregnancy, and family histories of CVD and hypertension compared with women without a HDP. No other meaningful differences were evident in other comorbidities, calendar year, or use of medications between the two groups.

A total of 997 women had incident CVD during 902,897 person-years of follow-up (Table 2). In women with HDP, the rate of subsequent CVD was two times higher ( $\mathrm{HR} 2.2,95 \% \mathrm{Cl} 1.7,2.7$ ) than in women with no history of HDP (Table 2). Although the point estimate from the timefixed analysis ( $\mathrm{HR} 2.1,95 \% \mathrm{Cl} 1.6,2.6$ ) was slightly lower than that of the MSM analysis, the overlapping Cls suggest no difference between the two.

During 869,531 person-years of follow-up, 6,812 women were diagnosed with hypertension or received a new prescription for an anti-hypertensive medication (Table 2). In women with a HDP, the rate of hypertension was 5 times that of women without $\mathrm{HDP}$ (HR 5.6, 95\% $\mathrm{Cl} 5.1$, 6.3). The time-fixed analysis produced a slightly lower estimate compared to the MSM analysis (HR 4.7, 95\% Cl 4.4, 5.0). In the time-fixed analyses for CVD and hypertension, none of the potential confounders were found to change the point estimate more than $10 \%$.

Our sensitivity analysis categorizing HDPs by level of severity suggests that women with other HDP have a higher risk of incident CVD compared with women without HDP (Table 2). For 
This is the peer reviewed version of the following article: [Grandi, S.M., Vallée-Pouliot, K., Reynier, P., Eberg, M., Platt, R.W., Arel, R., Basso, O., and Filion, K.B. (2017). Hypertensive Disorders in Pregnancy and the Risk of Subsequent Cardiovascular

women with a diagnosis of preeclampsia/eclampsia, the results are inconclusive due to the wide Cls. We found that both subgroups of HDP had an increased risk of hypertension. However, women with preeclampsia/eclampsia had a slightly higher risk compared to women with other HDP.

In sensitivity analyses that extended the exposure assessment window to 12 weeks postpartum, the point estimate for incident CVD was similar to the main analysis (Online Appendix 5). For hypertension, the point estimate and $95 \% \mathrm{Cl}$ from the sensitivity analysis were slightly lower than observed in our primary analysis (Online Appendix 5). The exclusion of women using cardiovascular medications in the year before their first pregnancy did not result in important differences (Online Appendix 6). Using age as the time scale in the Cox model did not impact the association of interest (HR 2.1, 95\% Cl 1.7, 2.6 and HR 4.7, 95\% Cl 4.4, 5.0 for CVD and hypertension, respectively). Finally, the analyses restricting the cohort to women with at least two pregnancies and a first unaffected pregnancy were consistent with the time-fixed analyses (Online Appendix 7).

\section{COMMENT}

\section{Main Findings}

This study was designed to evaluate the risk of incident CVD associated with HDP, accounting for time-varying confounding and intermediates. Women who experience HDP have an approximately doubling of the rate of incident CVD and a 5 times higher rate of hypertension. 
This is the peer reviewed version of the following article: [Grandi, S.M., Vallée-Pouliot, K., Reynier, P., Eberg, M., Platt, R.W., Arel, R., Basso, O., and Filion, K.B. (2017). Hypertensive Disorders in Pregnancy and the Risk of Subsequent Cardiovascular

Results stratified by type of HDP were inconclusive with respect to preeclampsia/eclampsia and subsequent CVD.

\section{Interpretation}

The minimal differences between the MSM and the time-fixed analyses in this study suggest that time-varying confounding does not play a major role in the association between HDP and subsequent CVD, potentially because the occurrence of HDP is a manifestation of an underlying increased risk of CVD. An alternative explanation may be that the risk of CVD is a consequence of HDP in the first pregnancy and HDP in subsequent pregnancies contribute minimally to this risk. ${ }^{3,21,22}$ However, the results of this study cannot discriminate between the relative contributions of these two underlying causes.

Previous studies have suggested that women with preeclampsia/eclampsia, primarily those with early onset preeclampsia (prior to 34 weeks), may be at higher risk of developing traditional risk factors for CVD and subsequent CVD. ${ }^{8,23-26}$ However, it remains unclear if preeclampsia is a contributing factor or an early marker of disease. The inconclusive findings in this study, with respect to women with preeclampsia/eclampsia, may in part be explained by the use of a GP database, which may be less likely to fully capture women followed by obstetricians. The small number of women in the subgroup with a diagnosis of preeclampsia/eclampsia in the cohort supports this hypothesis. These findings may also suggest that the CPRD may be capturing women with a less severe form of preeclampsia who deliver at term and have a similar risk of CVD to that of women with other forms of HDP. ${ }^{7}$ 
This is the peer reviewed version of the following article: [Grandi, S.M., Vallée-Pouliot, K., Reynier, P., Eberg, M., Platt, R.W., Arel, R., Basso, O., and Filion, K.B. (2017). Hypertensive Disorders in Pregnancy and the Risk of Subsequent Cardiovascular

The association between all types of HDP and subsequent risk of CVD and hypertension suggests that women with a history of HDP may warrant closer long-term follow-up to better manage risk factors for CVD. However, it is unclear whether HDP is a marker of an underlying risk or causally related to subsequent CVD. The nature of this association is obscured by common genetic and risk factors. ${ }^{27}$ It may also be that the stress incurred to the cardiovascular system during gestation may trigger a biological response that would otherwise not have occurred despite any genetic predisposition or risk factors for CVD.

\section{Strengths of the Study}

The main strength of this study is the comprehensive nature of the CPRD and the availability of numerous covariates often not available in administrative databases (i.e., BMI, smoking), which reduces the potential for residual confounding. Second, the use of a clinical database allows for an inclusive definition of both exposure and outcome based on diagnostic codes and clinical information, such as blood pressure readings, which limits the potential for misclassification. Finally, a MSM allows for the inclusion of more than one pregnancy per women and accounts for time-varying confounders. ${ }^{28}$

\section{Limitations of the Data}

One of the main limitations of this study is that gestational age is not readily available in the CPRD, resulting in potential misclassification of the gestational period and the timing of events based on its approximation. However, the algorithm used to estimate this period has been 
This is the peer reviewed version of the following article: [Grandi, S.M., Vallée-Pouliot, K., Reynier, P., Eberg, M., Platt, R.W., Arel, R., Basso, O., and Filion, K.B. (2017). Hypertensive Disorders in Pregnancy and the Risk of Subsequent Cardiovascular

previously validated ${ }^{17}$ and the addition of a 2-week grace period to the exposure definition likely minimized this problem. The CPRD involves GP records and, although GPs in the UK serve as the healthcare "gatekeepers", some pregnancies followed by midwives or obstetricians may not be recorded. Potentially missing women followed by midwives are unlikely to result in an important bias, as these women are likely to have a similar risk profile as those followed by GPs. However, it is possible that this may have resulted in selection bias and may influence the generalizability of the results. For example, the exclusion of women with severe forms of preeclampsia may result in incorrectly suggesting that these women have a similar risk profile as women with other forms of HDP. With over 20 years of follow-up information in the CPRD, there is the potential for misclassification due to changes in diagnostic criteria for HDP over the study period. Although the objective was to incorporate multiple pregnancies, the majority of women ( $n=101,878 ; 69.4 \%$ ) had only one pregnancy during the study period, limiting the assessment of the association of HDP over multiple pregnancies on later CVD. While most of the diagnostic and clinical information is well recorded in the CPRD, family or personal history variables may be subject to misclassification and may lead to residual confounding. In addition, some women may have undiagnosed prevalent hypertension at baseline masked by the decrease in blood pressure that occurs in the first and second trimesters. ${ }^{30,31}$ However, this is likely to be the case in only a small proportion of women in the study cohort, particularly given the exclusion of women with a diagnosis of hypertension or high blood pressure readings in the year prior to index pregnancy. 
This is the peer reviewed version of the following article: [Grandi, S.M., Vallée-Pouliot, K., Reynier, P., Eberg, M., Platt, R.W., Arel, R., Basso, O., and Filion, K.B. (2017). Hypertensive Disorders in Pregnancy and the Risk of Subsequent Cardiovascular

Misclassification of the outcome is possible, as several studies have suggested that hypertension in pregnancy may take longer than 6 weeks to resolve. ${ }^{20}$ Although sensitivity analyses using a 12-week window produced slightly attenuated results, the finding of a substantially increased risk of incident CVD and hypertension remained. Finally, previous studies have suggested that the risk of CVD varies according to parity, with increasing risk across the grand (5-7 babies) and great-grand ( $>7$ babies) multiparity groups. ${ }^{32}$ The categorization of parity as $1,2-4$, and $\geq 5$ may result in some residual confounding, but the small number of women with $>5$ pregnancies prevented further sub-classification of parity.

\section{CONCLUSIONS}

Women with a HDP are at increased risk of developing subsequent CVD. Similar estimates were obtained with the analysis accounting for time-varying confounding and cumulative pregnancies affected by HDP, and the time-fixed analysis, which assessed HDP in the first pregnancy only. This suggests that subsequent pregnancies do not confound the association between a first episode of HDP and later CVD. With their higher risk of CVD, women with a history of HDP may warrant closer long-term follow-up for early management of risk factors for CVD. 
This is the peer reviewed version of the following article: [Grandi, S.M., Vallée-Pouliot, K., Reynier, P., Eberg, M., Platt, R.W., Arel, R., Basso, O., and Filion, K.B. (2017). Hypertensive Disorders in Pregnancy and the Risk of Subsequent Cardiovascular

\section{ACKNOWLEDGEMENTS}

Funding/Support: Sonia Grandi is supported by a Doctoral Award from the Fonds de recherche du Québec - Santé (FRQS). Kristian Filion holds a Canadian Institutes of Health Research (CIHR) New Investigator award. This study was conducted using data acquired thanks to a CIHR operating grant (grant number MOP 126171). Robert Platt is a Chercheur-National from the FRQS, a member of the Research Institute of the McGill University Health Centre, which receives financial support from the FRQS, and holds the Albert Boehringer I endowed chair in Pharmacoepidemiology at McGill University. 
This is the peer reviewed version of the following article: [Grandi, S.M., Vallée-Pouliot, K., Reynier, P., Eberg, M., Platt, R.W., Arel, R., Basso, O., and Filion, K.B. (2017). Hypertensive Disorders in Pregnancy and the Risk of Subsequent Cardiovascular

\section{REFERENCES}

1. Kuklina EV, Ayala C, Callaghan WM. Hypertensive disorders and severe obstetric morbidity in the United States. Obstetrics and gynecology 2009;113:1299-306.

2. Mannisto $\mathrm{T}$, Mendola $\mathrm{P}$, Vaarasmaki $\mathrm{M}$, et al. Elevated blood pressure in pregnancy and subsequent chronic disease risk. Circulation 2013;127:681-90.

3. Vest AR, Cho LS. Hypertension in pregnancy. Current atherosclerosis reports 2014;16:395.

4. van Rijn BB, Nijdam ME, Bruinse HW, et al. Cardiovascular disease risk factors in women with a history of early-onset preeclampsia. Obstetrics and gynecology 2013;121:1040-8.

5. Valdiviezo C, Garovic VD, Ouyang P. Preeclampsia and hypertensive disease in pregnancy: their contributions to cardiovascular risk. Clinical cardiology 2012;35:160-5.

6. Powe CE, Levine RJ, Karumanchi SA. Preeclampsia, a disease of the maternal endothelium: the role of antiangiogenic factors and implications for later cardiovascular disease. Circulation 2011;123:2856-69.

7. Skjaerven R, Wilcox AJ, Klungsoyr K, et al. Cardiovascular mortality after pre-eclampsia in one child mothers: prospective, population based cohort study. BMJ 2012;345:e7677.

8. Irgens HU, Reisaeter L, Irgens LM, Lie RT. Long term mortality of mothers and fathers after pre-eclampsia: population based cohort study. BMJ 2001;323:1213-7.

9. Magnussen EB, Vatten $L$, Smith GD, Romundstad PR. Hypertensive disorders in pregnancy and subsequently measured cardiovascular risk factors. Obstetrics and gynecology 2009;114:961-70.

10. The Clinical Practice Research Datalink 2014. (Accessed 1st March, 2014, at http://www.cprd.com/intro.asp.)

11. Herrett E, Gallagher AM, Bhaskaran K, et al. Data Resource Profile: Clinical Practice Research Datalink (CPRD). Int J Epidemiol 2015;44:827-36.

12. Chisholm J. The Read clinical classification. BMJ 1990;300:1092.

13. Magee LA, Helewa M, Moutquin JM, von Dadelszen P, Hypertension Guideline C, Strategic Training Initiative in Research in the Reproductive Health Sciences S. Diagnosis, evaluation, and management of the hypertensive disorders of pregnancy. Journal of obstetrics and gynaecology Canada : JOGC = Journal d'obstetrique et gynecologie du Canada : JOGC 2008;30:S1-48. 
This is the peer reviewed version of the following article: [Grandi, S.M., Vallée-Pouliot, K., Reynier, P., Eberg, M., Platt, R.W., Arel, R., Basso, O., and Filion, K.B. (2017). Hypertensive Disorders in Pregnancy and the Risk of Subsequent Cardiovascular

14. Hypertension in Pregnancy: The Management of Hypertensive Disorders During Pregnancy: National Institute for Health and Clinical Excellence; January 2011. Report No.: NICE Clinical Guideline 107.

15. Eberg M PR, Filion KB. The Estimation of Gestational Age at Birth in Database Studies. Epidemiology 2017; In Press.

16. Jukic AM, Baird DD, Weinberg CR, McConnaughey DR, Wilcox AJ. Length of human pregnancy and contributors to its natural variation. Human reproduction 2013;28:284855.

17. Margulis AV, Setoguchi S, Mittleman MA, Glynn RJ, Dormuth CR, Hernandez-Diaz S. Algorithms to estimate the beginning of pregnancy in administrative databases. Pharmacoepidemiology and drug safety 2013;22:16-24.

18. Schafer JL. Multiple imputation: a primer. Stat Methods Med Res 1999;8:3-15.

19. Hernan MA, Brumback B, Robins JM. Marginal structural models to estimate the causal effect of zidovudine on the survival of HIV-positive men. Epidemiology 2000;11:561-70.

20. Mammaro A, Carrara S, Cavaliere A, et al. Hypertensive disorders of pregnancy. Journal of prenatal medicine 2009;3:1-5.

21. Saftlas AF, Levine RJ, Klebanoff MA, et al. Abortion, changed paternity, and risk of preeclampsia in nulliparous women. Am J Epidemiol 2003;157:1108-14.

22. The American College of Obstetrics and Gynecologists. Key Elements for the Management of Hypertensive Crisis in Pregnancy (In-Patient). Clinical Practice Guidelines 2013. (Accessed $1^{\text {st }}$ February 2016 at http://www.acog.org/ /media/Districts/District\%20Il/PDFs/Optimizing_Protocols_In_O B_HTN_Series_3-\%20Version\%201.pdf?dmc=1.)

23. Garovic VD, Bailey KR, Boerwinkle E, et al. Hypertension in pregnancy as a risk factor for cardiovascular disease later in life. J Hypertens 2010;28:826-33.

24. Wilson BJ, Watson MS, Prescott GJ, et al. Hypertensive diseases of pregnancy and risk of hypertension and stroke in later life: results from cohort study. BMJ 2003;326:845.

25. Smith GN, Pudwell J, Walker M, Wen SW. Ten-year, thirty-year, and lifetime cardiovascular disease risk estimates following a pregnancy complicated by preeclampsia. Journal of obstetrics and gynaecology Canada 2012;34:830-5.

26. Mongraw-Chaffin ML, Cirillo PM, Cohn BA. Preeclampsia and cardiovascular disease death: prospective evidence from the child health and development studies cohort. Hypertension 2010;56:166-71. 
This is the peer reviewed version of the following article: [Grandi, S.M., Vallée-Pouliot, K., Reynier, P., Eberg, M., Platt, R.W., Arel, R., Basso, O., and Filion, K.B. (2017). Hypertensive Disorders in Pregnancy and the Risk of Subsequent Cardiovascular

27. Ahmed R, Dunford J, Mehran R, Robson S, Kunadian V. Pre-eclampsia and future cardiovascular risk among women: a review. J Am Coll Cardiol 2014;63:1815-22.

28. Cole SR, Hernan MA. Constructing inverse probability weights for marginal structural models. Am J Epidemiol 2008;168:656-64.

29. Ghosh G, Grewal J, Mannisto T, et al. Racial/ethnic differences in pregnancy-related hypertensive disease in nulliparous women. Ethn Dis 2014;24:283-9.

30. Mahendru AA, Everett TR, Wilkinson IB, Lees CC, McEniery CM. A longitudinal study of maternal cardiovascular function from preconception to the postpartum period. J Hypertens 2014;32:849-56.

31. Mahendru AA, Everett TR, Wilkinson IB, Lees CC, McEniery CM. Maternal cardiovascular changes from pre-pregnancy to very early pregnancy. J Hypertens 2012;30:2168-72.

32. Babinszki A, Kerenyi T, Torok O, Grazi V, Lapinski RH, Berkowitz RL. Perinatal outcome in grand and great-grand multiparity: effects of parity on obstetric risk factors. American journal of obstetrics and gynecology 1999;181:669-74. 
This is the peer reviewed version of the following article: [Grandi, S.M., Vallée-Pouliot, K., Reynier, P., Eberg, M., Platt, R.W., Arel, R., Basso, O., and Filion, K.B. (2017). Hypertensive Disorders in Pregnancy and the Risk of Subsequent Cardiovascular

Table 1. Patient Characteristics at First Pregnancy by Hypertensive Disorders of Pregnancy Exposure Status.

\begin{tabular}{|c|c|c|}
\hline Characteristics & $\begin{array}{c}\text { Exposed } \\
(\mathrm{N}=\mathbf{5 , 3 9 9})\end{array}$ & 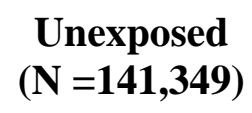 \\
\hline Age, mean (SD) & $29.8(6.0)$ & $29.2(6.1)$ \\
\hline \multicolumn{3}{|l|}{ BMI, n $(\%)$} \\
\hline Underweight $\left(<18.5 \mathrm{~kg} / \mathrm{m}^{2}\right)$ & $74(1.4)$ & $4,427(3.1)$ \\
\hline Normal $\left(18.5 \mathrm{~kg} / \mathrm{m}^{2}\right.$ to $\left.24.9 \mathrm{~kg} / \mathrm{m}^{2}\right)$ & $1,705(31.6)$ & $56,043(39.6)$ \\
\hline Overweight ( $25 \mathrm{~kg} / \mathrm{m}^{2}$ to $29.9 \mathrm{~kg} / \mathrm{m}^{2}$ ) & $988(18.3)$ & $20,009(14.2)$ \\
\hline Obese $\left(\geq 30.0 \mathrm{~kg} / \mathrm{m}^{2}\right)$ & $698(12.9)$ & $10,027(7.1)$ \\
\hline Unknown & $1,934(35.8)$ & $50,843(36.0)$ \\
\hline \multicolumn{3}{|l|}{ Smoking status, $\mathrm{n}(\%)^{*}$} \\
\hline Non-smoker & $2,693(49.9)$ & $65,297(46.2)$ \\
\hline Smoker & $1,982(36.7)$ & $56,423(39.9)$ \\
\hline Unknown & $724(13.4)$ & $19,629(13.9)$ \\
\hline Excessive alcohol use, $\mathrm{n}(\%)^{\dagger}$ & $176(3.3)$ & $4,023(2.9)$ \\
\hline Multiple gestations at first pregnancy, n (\%) & $20(0.4)$ & $396(0.3)$ \\
\hline Diabetes mellitus, n (\%) & $70(1.3)$ & $855(0.6)$ \\
\hline Gestational diabetes in first pregnancy, \% (n) & $63(1.2)$ & $942(0.7)$ \\
\hline Dyslipidemia, n (\%) & $20(0.4)$ & $267(0.2)$ \\
\hline Polycystic ovary syndrome, n (\%) & $209(3.9)$ & $4,628(3.3)$ \\
\hline Renal disease, n (\%) & $34(0.6)$ & $436(0.3)$ \\
\hline Venous thromboembolism, n (\%) & $72(1.3)$ & $1,302(0.9)$ \\
\hline History of migraine, $\mathrm{n}(\%)$ & $546(10.1)$ & $12,147(8.6)$ \\
\hline History of depression, $\mathrm{n}(\%)$ & $389(7.2)$ & $10,027(7.1)$ \\
\hline Sleep apnea, n (\%) & S & $107(0.1)$ \\
\hline Family history of cardiovascular disease, n (\%) & $732(13.6)$ & $16,456(11.6)$ \\
\hline Family history of hypertension, n (\%) & $479(8.9)$ & $9,036(6.4)$ \\
\hline $\begin{array}{l}\text { Number of distinct drug classes prescribed in the } \\
\text { previous year, mean (SD) }\end{array}$ & $2.9(2.8)$ & $2.7(2.7)$ \\
\hline
\end{tabular}


This is the peer reviewed version of the following article: [Grandi, S.M., Vallée-Pouliot, K., Reynier, P., Eberg, M., Platt, R.W., Arel, R., Basso, O., and Filion, K.B. (2017). Hypertensive Disorders in Pregnancy and the Risk of Subsequent Cardiovascular

Antidepressants

Aspirin

Anti-migraine

NSAIDs

Oral contraceptives

Statins
405 (7.5)

$15(0.3)$

$116(2.1)$

697 (12.9)

2,015 (37.3)

$\mathrm{S}$
10,644 (7.5)

$369(0.3)$

$2,772(2.0)$

17,083 (12.1)

53,563 (37.9)

$56(<0.1)$

$87(0.1)$

Abbreviations: BMI: body mass index; DBP: Diastolic blood pressure; IQR: inter-quartile range; NSAIDs: non-steroidal anti-inflammatory drugs; $S$ : data suppressed due to a cell count $\leq 5$ in accordance with CPRD privacy requirements; SBP: Systolic blood pressure. "The smoking category includes current and former smokers; 'Excess alcohol use is defined using diagnostic codes (Read codes) for excess alcohol use, for alcohol-related comorbidities, and for treatment for alcohol abuse. Including selective serotonin reuptake inhibitors (SSRIs), serotonin-norepinephrine reuptake inhibitors (SNRIs), and other antidepressants (including tricyclic antidepressants and monoamine oxidase inhibitors). 
This is the peer reviewed version of the following article: [Grandi, S.M., Vallée-Pouliot, K., Reynier, P., Eberg, M., Platt, R.W., Arel, R., Basso, O., and Filion, K.B. (2017). Hypertensive Disorders in Pregnancy and the Risk of Subsequent Cardiovascular

Table 2. Risk of Cardiovascular Disease and Hypertension or Prescription of Anti-Hypertensive Medications Among Women with Hypertensive Disorders in Pregnancy.

\begin{tabular}{|c|c|c|c|c|c|c|}
\hline & \multicolumn{5}{|c|}{ Time-Fixed Exposure Defined by First Pregnancy ${ }^{* \dagger}$} & \multirow{2}{*}{$\begin{array}{c}\text { Marginal Structural Model }^{\ddagger} \\
\text { Adjusted HR } \\
(95 \% \text { CI })\end{array}$} \\
\hline & Events & $\begin{array}{c}\text { Person- } \\
\text { Years }\end{array}$ & $\begin{array}{c}\text { Incidence Rate (95\% } \\
\text { CI) }\end{array}$ & $\begin{array}{c}\text { Crude HR (95\% } \\
\text { CI) }\end{array}$ & $\begin{array}{l}\text { Adjusted HR } \\
\text { (95\% CI) }\end{array}$ & \\
\hline \multicolumn{7}{|l|}{ Cardiovascular Disease } \\
\hline No Hypertensive Disorders & 918 & 868,988 & $1.1(1.0,1.1)$ & 1.0 (Ref) & $1.0(\mathrm{Ref})$ & $1.0(\mathrm{Ref})$ \\
\hline Hypertensive Disorders & 79 & 33,909 & $2.3(1.9,2.9)$ & $2.2(1.8,2.8)$ & $2.1(1.6,2.6)$ & $2.2(1.7,2.7)$ \\
\hline Preeclampsia/Eclampsia & $\mathrm{S}$ & $\mathrm{S}$ & $0.7(0.2,2.2)$ & $0.7(0.2,2.0)$ & $0.6(0.2,1.9)$ & $1.1(0.4,3.4)$ \\
\hline Other Gestational Hypertension & $\mathrm{S}$ & $\mathrm{S}$ & $2.6(2.1,3.2)$ & $2.4(1.9,3.1)$ & $2.3(1.8,2.9)$ & $2.3(1.8,3.0)$ \\
\hline \multicolumn{7}{|l|}{ Hypertension } \\
\hline No Hypertensive Disorders & 5,791 & 841,191 & $6.9(6.7,7.1)$ & 1.0 (Ref) & 1.0 (Ref) & 1.0 (Ref) \\
\hline Hypertensive Disorders & 1,021 & 28,340 & $36.0(33.9,38.3)$ & $5.2(4.9,5.6)$ & $4.7(4.4,5.0)$ & $5.6(5.1,6.3)$ \\
\hline Preeclampsia/Eclampsia & 133 & 3,502 & $38.0(32.0,45.0)$ & $5.5(4.6,6.5)$ & $5.2(4.3,6.1)$ & $6.5(5.2,8.1)$ \\
\hline Other Gestational Hypertension & 888 & 24,837 & $35.8(33.5,38.2)$ & $5.2(4.9,5.6)$ & $4.6(4.3,5.0)$ & $5.5(5.0,6.0)$ \\
\hline
\end{tabular}

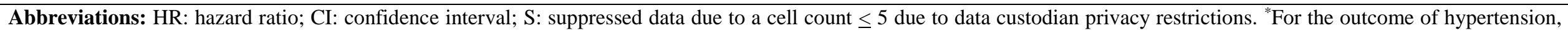

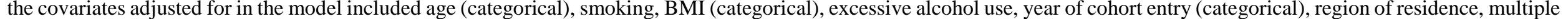

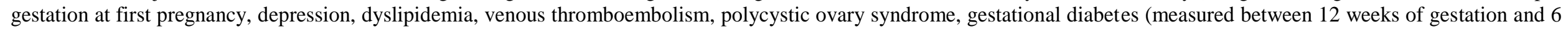

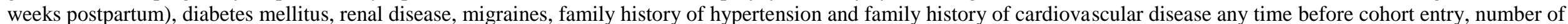

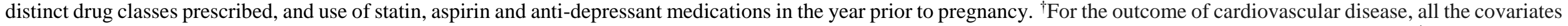

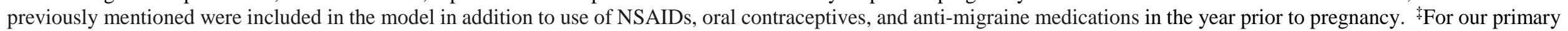

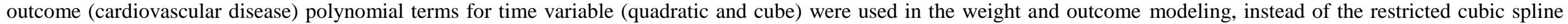
function. For our secondary outcome (hypertension), patients with extreme weights were excluded from the analyses. 
This is the peer reviewed version of the following article: [Grandi, S.M., Vallée-Pouliot, K., Reynier, P., Eberg, M., Platt, R.W., Arel, R., Basso, O., and Filion, K.B. (2017). Hypertensive Disorders in Pregnancy and the Risk of Subsequent Cardiovascular

Figure 1

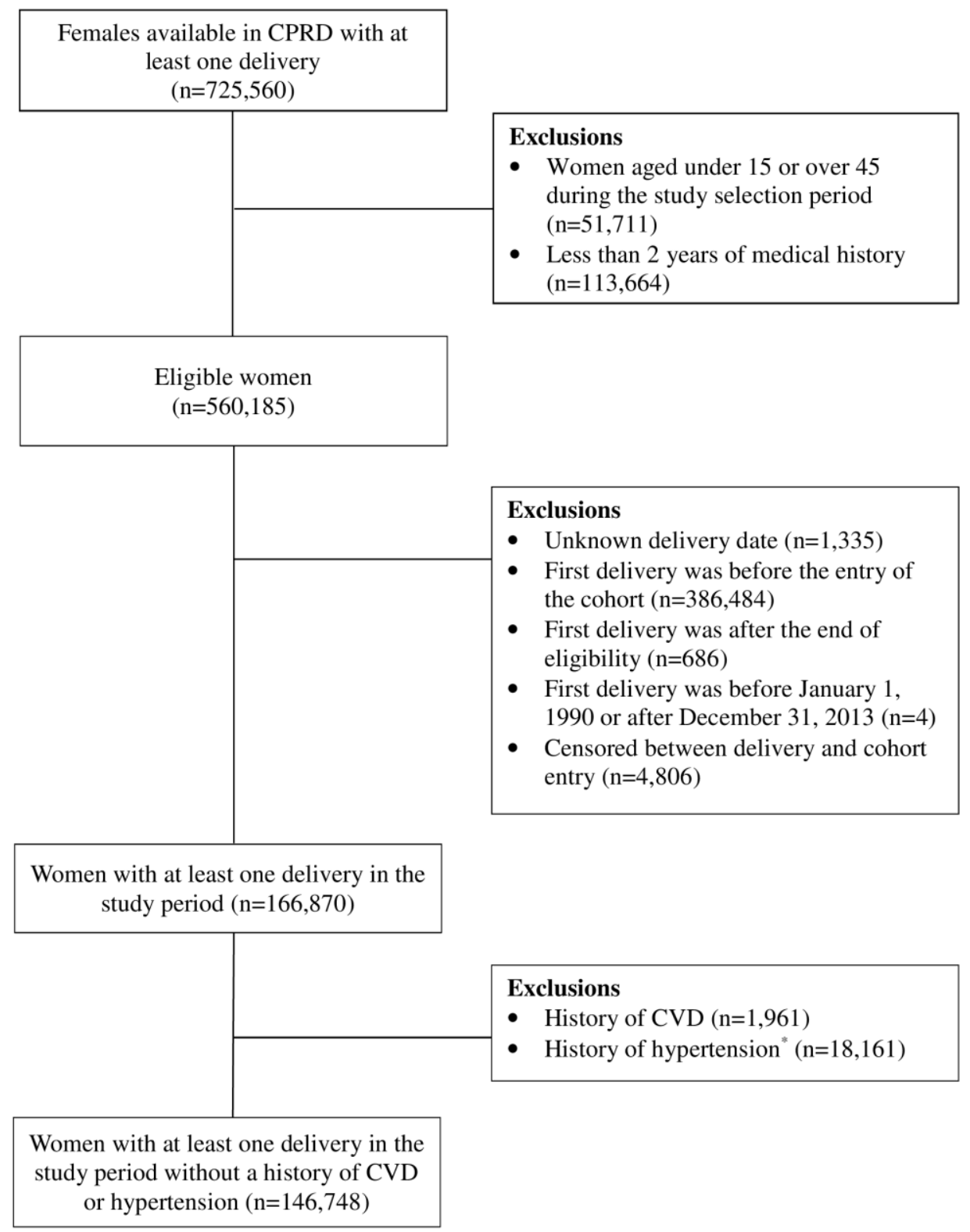


This is the peer reviewed version of the following article: [Grandi, S.M., Vallée-Pouliot, K., Reynier, P., Eberg, M., Platt, R.W., Arel, R., Basso, O., and Filion, K.B. (2017). Hypertensive Disorders in Pregnancy and the Risk of Subsequent Cardiovascular 
This is the peer reviewed version of the following article: [Grandi, S.M., Vallée-Pouliot, K., Reynier, P., Eberg, M., Platt, R.W., Arel, R., Basso, O., and Filion, K.B. (2017). Hypertensive Disorders in Pregnancy and the Risk of Subsequent Cardiovascular

Online Appendix 1. Definitions for the Primary and Secondary Outcomes and Covariate.

Primary Outcome - Cardiovascular Disease

- Cerebrovascular disease

- Coronary artery disease

- Coronary revascularization

- Myocardial infarction

- Peripheral arterial disease

- Transient Ischemic Attack

- Stroke

Secondary Outcome - Hypertension or Use of Anti-Hypertensive Medications

Anti-Hypertensive Medications

- Angiotensin-converting-enzyme (ACE) inhibitors

- Angiotensin II receptor antagonists (ARBs)

- Beta-blockers

- Calcium channel blockers

- Diuretics

\section{Covariate}

\section{Anti-Depressant Medications}

- Monoamine oxidase inhibitors

- Selective serotonin reuptake inhibitors (SNRIs)

- Serotonin-norepinephrine reuptake inhibitors (SSRIs)

- Tricyclic antidepressants 
This is the peer reviewed version of the following article: [Grandi, S.M., Vallée-Pouliot, K., Reynier, P., Eberg, M., Platt, R.W., Arel, R., Basso, O., and Filion, K.B. (2017). Hypertensive Disorders in Pregnancy and the Risk of Subsequent Cardiovascular

Online Appendix 2. Directed Acyclic Graph (DAG) Outlining the Temporality of the Association between Hypertensive Disorders in Pregnancy and Subsequent Cardiovascular Disease*

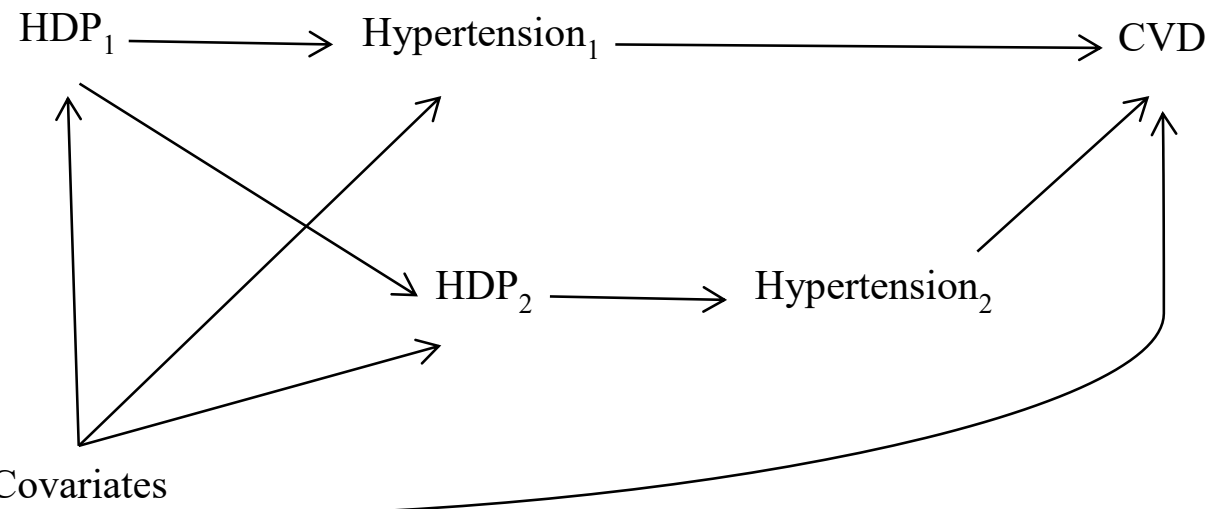

*This is a schematic DAG to illustrate the temporality of the association between hypertensive disorders in pregnancy and subsequent cardiovascular disease. This DAG does not reflect the complex nature of the true associations but rather is a simplistic overview of the underlying assumptions of our analyses. Abbreviations: CVD: Cardiovascular disease; HDP 1 : Hypertensive disorder in the $1^{\text {st }}$ pregnancy; $\mathrm{HDP}_{2}$ : Hypertensive disorder in subsequent pregnancies. 
This is the peer reviewed version of the following article: [Grandi, S.M., Vallée-Pouliot, K., Reynier, P., Eberg, M., Platt, R.W., Arel, R., Basso, O., and Filion, K.B. (2017). Hypertensive Disorders in Pregnancy and the Risk of Subsequent Cardiovascular

Online Appendix 3. Number of pregnancies per women during the follow-up period.

\begin{tabular}{|c|c|c|}
\hline $\begin{array}{l}\text { Number of pregnancies, } \\
n(\%)\end{array}$ & $\begin{array}{l}\text { Exposed } \\
(\mathrm{N}=5,399)\end{array}$ & $\begin{array}{l}\text { Unexposed } \\
(\mathrm{N}=141,349)\end{array}$ \\
\hline \multicolumn{3}{|l|}{ Cardiovascular Disease $^{*}$} \\
\hline 1 & $3,655(67.7)$ & $98,223(69.5)$ \\
\hline 2 & $1,482(27.5)$ & $36,081(25.5)$ \\
\hline 3 & $229(4.2)$ & $5,910(4.2)$ \\
\hline$\geq 4$ & $33(0.6)$ & $1,135(0.8)$ \\
\hline \multicolumn{3}{|l|}{ Hypertension ${ }^{\dagger}$} \\
\hline 1 & $3,828(70.9)$ & $98,689(69.8)$ \\
\hline 2 & $1,347(24.5)$ & $35,823(25.3)$ \\
\hline 3 & $196(3.6)$ & $5,747(4.1)$ \\
\hline$\geq 4$ & $28(0.5)$ & $1,090(0.8)$ \\
\hline \multicolumn{3}{|c|}{$\begin{array}{l}{ }^{*} \text { A total of } 200,365 \text { pregnancies during the follow-up period. }{ }^{\dagger} \text { A total of } 199,419 \text { pregnancies during the } \\
\text { follow-up period. } \\
\text { Note: The number of pregnancies differs for the primary and secondary outcome since the duration of } \\
\text { follow-up differs for each outcome. }\end{array}$} \\
\hline
\end{tabular}


This is the peer reviewed version of the following article: [Grandi, S.M., Vallée-Pouliot, K., Reynier, P., Eberg, M., Platt, R.W., Arel, R., Basso, O., and Filion, K.B. (2017). Hypertensive Disorders in Pregnancy and the Risk of Subsequent Cardiovascular

Online Appendix 4. Number of exposed pregnancies during the follow-up period.

\section{A) For the primary outcome of CVD.}

\begin{tabular}{|l|c|c|c|c|}
\hline & \multicolumn{4}{|c|}{ Total Number of Exposed Pregnancies } \\
\hline Total Number of Pregnancies, $\mathbf{n}(\boldsymbol{\%})$ & $\mathbf{1}$ & $\mathbf{2}$ & $\mathbf{\geq}$ & Total \\
\hline 1 & $3,655(56.8)$ & $0(0.0)$ & $0(0.0)$ & 3,655 \\
\hline 2 & $2,209(34.3)$ & $276(70.1)$ & $0(0.0)$ & 2,485 \\
\hline$\geq 3$ & $569(8.8)$ & $118(29.9)$ & $27(100.0)$ & 714 \\
\hline Total & 6,433 & 394 & 27 & 6,854 \\
\hline
\end{tabular}

B) For the secondary outcome of hypertension.

\begin{tabular}{|l|c|c|c|c|}
\hline & \multicolumn{4}{|c|}{ Total Number of Exposed Pregnancies } \\
\hline Number of Pregnancies, $\mathbf{n}(\boldsymbol{\%})$ & $\mathbf{1}$ & $\mathbf{2}$ & $\geq \mathbf{3}$ & Total \\
\hline 1 & $3,828(60.4)$ & $0(0.0)$ & $0(0.0)$ & 3,828 \\
\hline 2 & $2,035(32.1)$ & $225(74.5)$ & $0(0.0)$ & 2,260 \\
\hline$\geq 3$ & $472(7.5)$ & $77(25.5)$ & $14(100.0)$ & 563 \\
\hline Total & 6,335 & 302 & 14 & 6,651 \\
\hline
\end{tabular}


This is the peer reviewed version of the following article: [Grandi, S.M., Vallée-Pouliot, K., Reynier, P., Eberg, M., Platt, R.W., Arel, R., Basso, O., and Filion, K.B. (2017). Hypertensive Disorders in Pregnancy and the Risk of Subsequent Cardiovascular

Online Appendix 5. Sensitivity analyses with the exposure definition period from 18 weeks of gestation to 12 weeks post-partum.

\begin{tabular}{|c|c|c|c|c|c|c|}
\hline Exposure & Events & $\begin{array}{l}\text { Person- } \\
\text { Years }\end{array}$ & $\begin{array}{c}\text { Incidence Rate } \\
(95 \% \mathrm{CI})\end{array}$ & $\begin{array}{c}\text { Time-Fixed Approach: } \\
\text { Crude HR (95\% CI) }\end{array}$ & $\begin{array}{l}\text { Time-Fixed Approach: } \\
\text { Adjusted HR (95\% CI) }\end{array}$ & $\begin{array}{c}\text { MSM: } \\
\text { HR }(95 \% \text { CI })\end{array}$ \\
\hline \multicolumn{7}{|c|}{ Cardiovascular Disease ${ }^{*}$} \\
\hline Unexposed & 867 & 849,169 & $1.0(1.0,1.1)$ & 1.0 (Ref) & 1.0 (Ref) & 1.0 (Ref) \\
\hline Exposed & 75 & 37,122 & $2.0(1.6,2.5)$ & $2.0(1.6,2.5)$ & $1.8(1.5,2.3)$ & $2.0(1.6,2.5)$ \\
\hline \multicolumn{7}{|c|}{ Hypertension $^{\dagger}$} \\
\hline Exposed & 873 & 32,160 & $27.2(25.4,29.0)$ & $4.1(3.8,4.4)$ & $3.7(3.4,4.0)$ & $4.8(4.3,5.4)$ \\
\hline \multicolumn{7}{|c|}{$\begin{array}{l}\text { Abbreviations: CI: Confidence interval; HR: Hazard ratio; "Time-fixed analyses included time-fixed covariates included age (categorical), smoking, } \\
\text { obesity, excessive alcohol use, year of cohort entry (categorical), region of residence, multiple gestation at first pregnancy, depression, dyslipidemia, } \\
\text { venous thromboembolism, polycystic ovary syndrome, gestational diabetes (measured between } 12 \text { weeks of gestation and } 6 \text { weeks postpartum), } \\
\text { diabetes mellitus, renal disease, migraines, family history of hypertension and family history of cardiovascular disease any time before cohort entry, } \\
\text { number of distinct drug classes prescribed, and use of statins, aspirin, NSAIDs, oral contraceptives, anti-migraine medication, anti-depressants in the } \\
\text { year prior to pregnancy. }{ }^{\dagger} \text { Time-fixed analyses included time-fixed covariates included those previously described for the primary outcome with the } \\
\text { exception of prior use of NSAIDS, oral contraceptives, and use of anti-migraine medications. }\end{array}$} \\
\hline
\end{tabular}


This is the peer reviewed version of the following article: [Grandi, S.M., Vallée-Pouliot, K., Reynier, P., Eberg, M., Platt, R.W., Arel, R., Basso, O., and Filion, K.B. (2017). Hypertensive Disorders in Pregnancy and the Risk of Subsequent Cardiovascular

Online Appendix 6. Time-fixed analyses excluding patients with a prescription of cardiovascular medications in the year prior to the first pregnancy and up to cohort entry and censoring women who took these medications after cohort entry.

\begin{tabular}{|c|c|c|c|c|c|c|}
\hline & \multicolumn{3}{|c|}{ Cardiovascular Disease } & \multicolumn{3}{|c|}{ Hypertension } \\
\hline $\begin{array}{l}\text { Cardiovascular } \\
\text { Medication }\end{array}$ & Events & $\begin{array}{c}\text { Crude HR } \\
(95 \% \text { CI })\end{array}$ & $\begin{array}{c}\text { Adjusted HR } \\
(95 \% \mathrm{CI})\end{array}$ & Events & $\begin{array}{c}\text { Crude HR } \\
(95 \% \text { CI })\end{array}$ & $\begin{array}{c}\text { Adjusted HR } \\
(95 \% \mathrm{CI})\end{array}$ \\
\hline \multicolumn{7}{|l|}{ Statins ${ }^{*}$} \\
\hline No HDP & 879 & 1.00 (Ref) & $1.00($ Ref $)$ & 5,692 & 1.00 (Ref) & 1.00 (Ref) \\
\hline HDP & 69 & $2.0(1.6,2.6)$ & $1.9(1.5,2.5)$ & 1,011 & $5.3(4.9,5.7)$ & $4.6(4.3,5.0)$ \\
\hline \multicolumn{7}{|l|}{ Warfarin } \\
\hline No HDP & 901 & 1.00 (Ref) & $1.00($ Ref $)$ & 5,740 & 1.00 (Ref) & 1.00 (Ref) \\
\hline HDP & 76 & $2.2(1.7,2.7)$ & $2.0(1.6,2.6)$ & 1,014 & $5.3(4.9,5.6)$ & $4.6(4.3,4.9)$ \\
\hline \multicolumn{7}{|l|}{ Fibrates } \\
\hline No HDP & 917 & 1.00 (Ref) & $1.00($ Ref $)$ & 5,787 & $1.00($ Ref $)$ & 1.00 (Ref) \\
\hline HDP & 78 & $2.2(1.7,2.8)$ & $2.0(1.6,2.6)$ & 1,020 & $5.2(4.9,5.6)$ & $4.6(4.3,4.9)$ \\
\hline \multicolumn{7}{|l|}{ Aspirin $^{\S}$} \\
\hline No HDP & 865 & 1.00 (Ref) & 1.00 (Ref) & 5,573 & 1.00 (Ref) & 1.00 (Ref) \\
\hline HDP & 68 & $2.1(1.6,2.6)$ & $1.9(1.5,2.5)$ & 995 & $5.4(5.1,5.8)$ & $4.7(4.4,5.1)$ \\
\hline \multicolumn{7}{|l|}{ All of the abovell } \\
\hline No HDP & 830 & $1.00($ Ref $)$ & $1.00($ Ref $)$ & 5,460 & 1.00 (Ref) & 1.00 (Ref) \\
\hline HDP & 62 & $2.0(1.5,2.6)$ & $1.9(1.4,2.4)$ & 981 & $5.5(5.1,5.8)$ & $4.8(4.5,5.1)$ \\
\hline
\end{tabular}


This is the peer reviewed version of the following article: [Grandi, S.M., Vallée-Pouliot, K., Reynier, P., Eberg, M., Platt, R.W., Arel, R., Basso, O., and Filion, K.B. (2017). Hypertensive Disorders in Pregnancy and the Risk of Subsequent Cardiovascular

Online Appendix 7. Risk of Cardiovascular Disease and Hypertension or Prescription of Anti-Hypertensive Medications Among Women with $\geq 2$ Pregnancies and a first pregnancy that was not affected by a hypertensive disorder of pregnancy.

\begin{tabular}{|c|c|c|c|c|c|}
\hline & \multicolumn{5}{|c|}{ Time-Fixed Exposure Defined by First Pregnancy $* \dagger$} \\
\hline & Events & Person-Years & $\begin{array}{c}\text { Incidence Rate } \\
(95 \% \mathrm{CI})\end{array}$ & $\begin{array}{r}\text { Crude HR } \\
(95 \% \text { CI })\end{array}$ & $\begin{array}{c}\text { Adjusted HR } \\
(95 \% \text { CI })\end{array}$ \\
\hline Cardiovascular Disease & & & & & \\
\hline No hypertensive Disorders & 213 & 256,783 & $0.8(0.7-1.0)$ & 1.0 (Ref) & $1.0(\mathrm{Ref})$ \\
\hline Hypertensive Disorders & 11 & 5,751 & $1.9(1.1-3.5)$ & $2.3(1.3-4.2)$ & $2.1(1.1-3.8)$ \\
\hline Hypertension & & & & & \\
\hline No Hypertensive Disorders & 1,569 & 247,239 & $6.4(6.0-6.7)$ & 1.0 (Ref) & 1.0 (Ref) \\
\hline Hypertensive Disorders & 180 & 4,327 & $41.6(35.9-48.1)$ & $6.6(5.6-7.7)$ & $5.7(4.8-6.6)$ \\
\hline
\end{tabular}

Abbreviations: HR: hazard ratio; CI: confidence interval. *For the outcome of hypertension, the covariates adjusted for in the model included age (categorical), smoking, BMI (categorical), excessive alcohol use, year of cohort entry (categorical), region of residence, multiple gestation at first pregnancy, depression, dyslipidemia, venous thromboembolism, polycystic ovary syndrome, gestational diabetes (measured between 12 weeks of gestation and 6 weeks postpartum), diabetes mellitus, renal disease, migraines, family history of hypertension and family history of cardiovascular disease any time before cohort entry, number of distinct drug classes prescribed, and use of statin, aspirin and anti-depressant medications in the year prior to pregnancy. †For the outcome of cardiovascular disease, all the covariates previously mentioned were included in the model in addition to use of NSAIDs, oral contraceptive, and anti-migraine medications in the year prior to pregnancy. 